\title{
Two-Point Correlation for Rich Clusters of Galaxies
}

\author{
S. Otto, H. David Politzer, and Mark B. Wise \\ California Institute of Technology, Pasadena, California 91125 \\ (Received 27 February 1986)
}

\begin{abstract}
The rich-cluster two-point correlation function is examined in a model where rich clusters of galaxies form at high peaks of primordial Gaussian mass-density fluctuations (averaged over a suitable volume). Particular attention is paid to the case when the primordial fluctuations have a Zel'dovich spectrum.

PACS numbers: $98.50 . \mathrm{Kg}, 98.80 .-\mathrm{k}$
\end{abstract}

Rich clusters of galaxies are observed to be strongly correlated at distances where the correlations of individual galaxies appear to be negligible. ${ }^{1}$ For example, the two-point correlation function for Abell clusters (richness $R>1$ ) is unity at about $25 h^{-1} \mathrm{Mpc}$, where $h$ is the Hubble constant and Mpc denotes megaparsecs, while the two-point correlation function for galaxies is unity at about $5 h^{-1} \mathrm{Mpc}$. In addition, the correlations of Abell clusters increase with richness. Kaiser has suggested that the origin of this enhancement is essentially statistical. ${ }^{2}$ On the assumption that rich clusters of galaxies arise wherever suitably averaged (filtered) Gaussian primordial mass-density fluctuations are above a high threshold, the $n$-point correlation functions of rich clusters can be expressed in terms of the two-point correlation function for the filtered mass-density fluctuations. ${ }^{2,3}$ The resulting richcluster two-point correlation function is enhanced over that of the filtered mass-density fluctuations, and, furthermore, this enhancement gets larger as the threshold is increased. This model for rich clusters, while reasonable for a universe dominated by cold, dark matter where hierarchical clustering occurs, would not be acceptable if rich clusters arose from the fragmentation of larger objects, as would occur if the universe were dominated by hot, dark matter.

Demanding that the filtered mass-density fluctuations exceed a given threshold is a constraint on their distribution that does not involve any derivatives. Consequently, in this model, zeros of the two-point correlation function for rich clusters must coincide with those of the two-point correlation function for the filtered mass-density fluctuations. Theoretical prejudices favor an $\Omega=1$ universe with primordial massdensity fluctuations that have a Zel'dovich power spectrum. With cold, dark matter, the power spectrum of the mass-density fluctuations falls off at large wave numbers, $k$, as $\ln (k) / k^{3}$ because fluctuations that entered the horizon in the radiation-dominated era only grow logarithmically prior to matter domination. In linear perturbation theory, this results in a (filtered) two-point correlation function for the mass-density fluctuations that crosses zero at about $17 h^{-2} \mathrm{Mpc}$.
If the filtered mass-density fluctuation field is above the rich-cluster threshold at one point, it is likely to be above at a neighboring point. In general, one does want to associate separate rich clusters with such points. A simple modification of Kaiser's original model which removes this difficulty, in a physically reasonable fashion, is to associate rich clusters only with local maxima (peaks) of the filtered mass-density fluctuations that are above the threshold. ${ }^{4,5}$ Because the peak constraint on the probability distribution for the filtered mass-density fluctuations involves derivatives of the mass-density field, zeros of the rich-cluster two-point correlation function no longer coincide with those of the filtered mass-density fluctuations. In removing close pairs of rich clusters, the peak condition introduces an anticorrelation. Hence, it is expected that the rich-cluster two-point correlation function will now cross zero before the two-point correlation of the filtered mass-density fluctuations. In the limit of large separation, the two-point correlation function of peaks is identical to the correlation function of all points above a high threshold; in this limit, these cases differ only in their densities, which are divided out of the dimensionless correlation functions.

The purpose of this Letter is to study the richcluster two-point correlation function in the model where rich clusters occur at high peaks of suitably averaged Gaussian primordial mass-density fluctuations. Particular attention will be paid to the distance out to which there is a significant correlation for rich clusters when the primordial mass-density fluctuations have a Zel'dovich power spectrum. ${ }^{6}$ Our results may present a problem for this model since the rich-cluster two-point correlation function is observed to be significant at quite large distances.

For Gaussian statistics, the probability that the filtered primordial mass-density fluctuations take the value $\boldsymbol{\epsilon}(\mathbf{x})$ is

$$
P[\boldsymbol{\epsilon}(\mathbf{x})]=Z^{-1} \exp \left[-\frac{1}{2} \int d^{3} x \epsilon(\mathbf{x}) K \epsilon(\mathbf{x})\right] .
$$

In Eq. (1), $Z$ is a normalization factor and $K$ is an operator that is diagonal in "wave-number space." 
Since the two-point correlation of the filtered mass-density fluctuations is

$$
\left\langle\epsilon\left(x_{1}\right) \epsilon\left(x_{2}\right)\right\rangle=\xi_{\rho}\left(\mathbf{x}_{1}-\mathbf{x}_{2}\right)=\int \frac{d^{3} k}{(2 \pi)^{3}} \frac{e^{i \mathbf{k} \cdot\left(\mathbf{x}_{1}-\mathbf{x}_{2}\right)}}{K(k)},
$$

$K(k)$ is the inverse of the power spectrum.

On the assumption that rich clusters occur at local maxima of $\epsilon(\mathbf{x})$ that are above the large threshold $t=\left[A \xi_{\rho}(0)\right]^{1 / 2}$, the probability $P_{N}\left(\mathbf{r}_{1}, \ldots, \mathbf{r}_{N}\right) d^{3} r_{1} \cdots d^{3} r_{N}$ of finding rich clusters in the volumes $d^{3} r_{1}, \ldots, d^{3} r_{N}$ about the points $\mathbf{r}_{1}, \ldots, \mathbf{r}_{N}$ is given by the functional integral

$$
P\left(\mathbf{r}_{1}, \ldots, \mathbf{r}_{N}\right)=\int[d \boldsymbol{\epsilon}(\mathbf{r})] P[\boldsymbol{\epsilon}(\mathbf{r})] \prod_{j=1}^{N}\left\{\int d \omega_{(j)}\left|\operatorname{det} \omega_{(j)}\right| \delta^{3}\left(\nabla \boldsymbol{\epsilon}\left(\mathbf{r}_{j}\right)\right) \delta^{6}\left(\nabla \nabla \epsilon\left(r_{j}\right)-\omega_{(j)}\right) \theta\left(\epsilon\left(\mathbf{r}_{j}\right)-t\right)\right\} .
$$

In Eq. (3), $d \omega_{(j)}$ is integrated over symmetric $3 \times 3$ matrices with positive definite eigenvalues. It is straightforward to evaluate $P_{N}\left(\mathrm{r}_{1}, \ldots, \mathrm{r}_{N}\right)$ in the limit of a large threshold $t$. We find that, to leading order in the threshold,

$$
P_{N}\left(\mathrm{r}_{1}, \ldots, \mathrm{r}_{N}\right) \simeq\left(\frac{1}{(2 \pi)^{2 N}(\operatorname{det} X)^{1 / 2}}\right)\left(\frac{\xi_{\rho}(0)}{t}\right)^{N} \exp \left(-\frac{1}{2} n^{T} X^{-1} n\right) \prod_{j=1}^{N}\left|\operatorname{det} W^{(j)}\right| .
$$

In Eq. (4), $X$ is the $4 N \times 4 N$ matrix with components

$$
[X]_{k l}=\left\langle y_{k} y_{l}\right\rangle \text {, }
$$

where $y_{k}$ are the components of the $4 N$-tuple $\left(\epsilon\left(\mathbf{r}_{1}\right), \ldots, \epsilon\left(\mathbf{r}_{N}\right), \nabla \epsilon\left(\mathbf{r}_{1}\right), \ldots, \nabla \epsilon\left(\mathbf{r}_{N}\right)\right) ; n$ is the $4 N$-tuple with components

$$
n_{k}=\left\{\begin{array}{l}
t \text { for } k=1, \ldots, N, \\
0 \text { for } k=N+1, \ldots, 4 N,
\end{array}\right.
$$

and $W^{(j)}$ is the symmetric $3 \times 3$ matrix with components

$$
\left[W^{(j)}\right]_{p q}=\sum_{k=1}^{4 N} \sum_{l=1}^{4 N}\left\langle y_{k} \nabla_{p} \nabla_{q} \epsilon\left(\mathbf{r}_{j}\right)\right\rangle\left[X^{-1}\right]_{k l} n_{l} .
$$

The $N$-point rich-cluster correlation function is given by

$$
1+\xi_{\mathrm{rc}}\left(\mathbf{x}_{1}, \ldots, \mathbf{x}_{N}\right)=\left(1 / P_{1}^{N}\right) P_{N}\left(\mathbf{x}_{1}, \ldots, \mathbf{x}_{N}\right) ;
$$

so Eqs. (4) - (7) determine (to leading order in the threshold) the rich-cluster correlations in terms of the twopoint correlation of the filtered mass-density fluctuations (and its first and second derivatives). According to Eqs. (4) - (7), the number density of rich clusters of galaxies $P_{1}$ is

$$
P_{1}-\frac{A}{(2 \pi)^{2}}\left[-\frac{\nabla^{2} \xi_{\rho}(0)}{3 \xi_{\rho}(0)}\right]^{3 / 2} \exp \left(-\frac{1}{2} A\right)
$$

We are particularly interested in the rich-cluster two-point correlation. Expanding it in powers of $\xi_{\boldsymbol{\rho}}(\mathbf{x}) / \xi_{\boldsymbol{\rho}}(0)$ and in powers of derivatives of $\xi_{\rho}(\mathbf{x})$ divided by derivatives of $\xi_{\rho}$ evaluated at $\mathbf{x}=0$ gives $^{8}$

$$
1+\xi_{\mathrm{rc}}(\mathbf{x}) \simeq\left\{1-\frac{6 \xi_{\boldsymbol{\rho}}(\mathbf{x})}{\xi_{\boldsymbol{\rho}}(0)}+\frac{6 \nabla^{2} \xi_{\boldsymbol{\rho}}(\mathbf{x})}{\nabla^{2} \xi_{\boldsymbol{\rho}}(0)}\right\} \exp \left[A \frac{\xi_{\boldsymbol{\rho}}(\mathbf{x})}{\xi_{\boldsymbol{\rho}}(0)}-A\left(\frac{\xi_{\rho}(\mathbf{x})}{\xi_{\rho}(0)}\right)^{2}+3 A \frac{\nabla \xi_{\rho}(\mathbf{x}) \cdot \nabla \xi_{\boldsymbol{\rho}}(\mathbf{x})}{\xi_{\boldsymbol{\rho}}(0) \nabla^{2} \xi_{\rho}(0)}\right] .
$$

The large coefficients of the terms in curly braces (which come from expanding the determinants of $W^{(j)}$ to linear order) indicate that such an expansion will typically not be reliable at the moderate distances $\left[\sim(10-40) h^{-1} \mathrm{Mpc}\right]$ of interest. ${ }^{4}$ Because of this feature and because we do not want to neglect terms subdominant in the threshold, we have also used a numerical Monte Carlo simulation to determine the rich-cluster twopoint correlation function. In Ref. 3, a simple expression was given for the rich-cluster reduced three-point correlation function in terms of the rich-cluster two-point correlation function-all in the model of counting points above threshold. Including the restriction to peaks, we now find that the large first corrections to that relation, 
which come from expanding the determinants of $W^{(j)}$ to linear order, all cancel, leaving

$$
\begin{aligned}
& 1+\xi_{\mathrm{rc}}\left(\mathbf{x}_{1}, \mathbf{x}_{2}, \mathbf{x}_{3}\right) \simeq\left[1+\xi_{\mathrm{rc}}\left(\mathbf{x}_{1}, \mathbf{x}_{2}\right)\right]\left[1+\xi_{\mathrm{rc}}\left(\mathbf{x}_{1}, \mathbf{x}_{3}\right)\right]\left[1-\xi_{\mathrm{rc}}\left(\mathbf{x}_{2}, \mathbf{x}_{3}\right)\right] \\
& \times \exp \left\{\frac{3 A \nabla \xi_{\boldsymbol{\rho}}\left(\mathbf{x}_{1}, \mathbf{x}_{2}\right) \cdot \nabla \xi\left(\mathbf{x}_{3}, \mathbf{x}_{2}\right)}{\xi_{\boldsymbol{\rho}}(0) \nabla^{2} \xi_{\boldsymbol{\rho}}(0)}+\frac{3 A \nabla \xi_{\boldsymbol{\rho}}\left(\mathbf{x}_{2}, \mathbf{x}_{3}\right) \cdot \nabla \xi_{\boldsymbol{\rho}}\left(\mathbf{x}_{1}, \mathbf{x}_{3}\right)}{\xi_{\boldsymbol{\rho}}(0) \nabla^{2} \xi_{\boldsymbol{\rho}}(0)}\right. \\
& +\frac{3 A \nabla \xi_{\boldsymbol{\rho}}\left(\mathbf{x}_{3}, \mathbf{x}_{1}\right) \cdot \nabla \xi_{\rho}\left(\mathbf{x}_{2}, \mathbf{x}_{1}\right)}{\xi_{\boldsymbol{\rho}}(0) \nabla^{2} \xi_{\boldsymbol{\rho}}(0)}-\frac{A \xi_{\boldsymbol{\rho}}\left(\mathbf{x}_{1}, \mathbf{x}_{2}\right) \xi_{\boldsymbol{\rho}}\left(\mathbf{x}_{2}, \mathbf{x}_{3}\right)}{\xi_{\boldsymbol{\rho}}(0)^{2}} \\
& \left.-\frac{A \xi_{\rho}\left(\mathbf{x}_{1}, \mathbf{x}_{3}\right) \xi_{\rho}\left(\mathbf{x}_{2}, \mathbf{x}_{3}\right)}{\xi_{\rho}(0)^{2}}-\frac{A \xi_{\rho}\left(\mathbf{x}_{1}, \mathbf{x}_{3}\right) \xi_{\rho}\left(\mathbf{x}_{2}, \mathbf{x}_{2}\right)}{\xi_{\rho}(0)^{2}}\right\} \text {. }
\end{aligned}
$$

To determine numerically the rich-cluster two-point correlation, we generated the filtered mass-density field $\epsilon(x)$ on $64^{3}$ cubic lattices with periodic boundary conditions and located the rich clusters at the local maxima of $\epsilon(\mathbf{x})$ above a threshold $t$. The lattice spacing was taken to be $2 h^{-1} \mathrm{Mpc}$, so that each lattice was $128 h^{-1} \mathrm{Mpc}$ across. The Fourier components of $\epsilon(\mathbf{x})$ were chosen according to a Gaussian probability distribution with power spectrum

$$
\tilde{\xi}_{\rho}(k) \propto k e^{-k^{2} R^{2} / 2} A^{2}(k),
$$

where

$$
A(k)=\frac{1}{\left[1+1.7 k / h^{2}+\left(4.3 k / h^{2}\right)^{3 / 2}+\left(k / h^{2}\right)^{2}\right]},
$$

when $k$ is measured in inverse megaparsecs. This is the power spectrum appropriate to cold, dark matter in an $\Omega=1$ universe with a Zel'dovich spectrum of mass-density fluctuations. ${ }^{9} R$ is the averaging radius. In the spherical model, the threshold $t$ determines the time at which the rich clusters collapsed. We used $t=1.7$, which corresponds in the spherical model to recently collapsed rich clusters. The averaging radius determines the typical mass of the rich clusters. Finally, the normalization of the power spectrum is adjusted to get the number density of rich clusters to be about $1 /\left(50 h^{-1} \mathrm{Mpc}\right)^{3}$.

Figure 1 shows the rich-cluster two-point correlation function which follows from an averaging radius of $7 h^{-1} \mathrm{Mpc}$ and a Hubble constant corresponding to $h=\frac{1}{2}$. Also shown in Fig. 1 (the dotted curve) is the leading term in the expansion of Eq. (10) for the richcluster two-point correlation \{i.e., $\exp \left[a \xi_{\rho}(x) /\right.$ $\left.\left.\xi_{\rho}(0)\right]-1\right\}$. This function crosses zero at the same point as the underlying density fluctuations, $\sim 34 h^{-1}$ $\mathrm{Mpc}$, while the rich-cluster two-point correlation function appears to cross zero at only about $26 h^{-1} \mathrm{Mpc}$. The solid curve shown in Fig. 1 is the mass-density correlation function $\xi_{\rho}(x)$ that follows from the power spectrum given in Eqs. (12). We have also used the Monte Carlo simulation to measure the two-point correlation of the $\epsilon(\mathbf{x})$ and of places where $\epsilon(\mathbf{x})$ is above the threshold $t$. These measurements agree quite well with the solid and dotted curves in Fig. 1, indicating that the difference in zero crossings for $\xi_{\boldsymbol{\rho}}(\mathbf{x})$ and $\xi_{\mathrm{rc}}(\mathbf{x})$ is not a lattice artifact.

The measured number density for rich clusters agrees at the $20 \%$ level with the high-threshold prediction given in Eq. (9). This suggests that for rich clusters of galaxies the threshold may be high enough for Eqs. (4) - (8) to give a reasonable approximation to their correlation functions.

We have explored the dependence of the richcluster two-point correlation function on the averaging radius $R$. Figure 2 is similar to Fig. 1 except that the averaging radius $R$ was chosen to be $9 h^{-1}$ Mpc.

The model for rich clusters used in this paper has also been applied to galaxies in an effort to reconcile virial studies which suggest $\Omega=0.2$ with theoretical

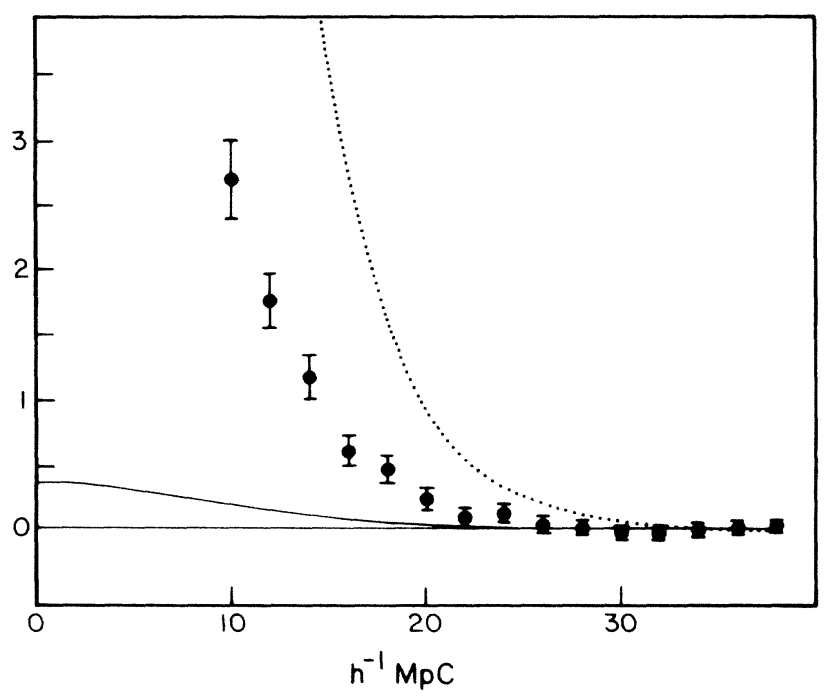

FIG. 1. Rich-cluster two-point correlation function with averaging radius $7 h^{-1} \mathrm{Mpc}$ and Hubble constant $h=\frac{1}{2}$. The dotted curve is the leading term in the expansion of Eq. (10) and the solid curve is the two-point correlation function for the filtered mass-density fluctuations. 


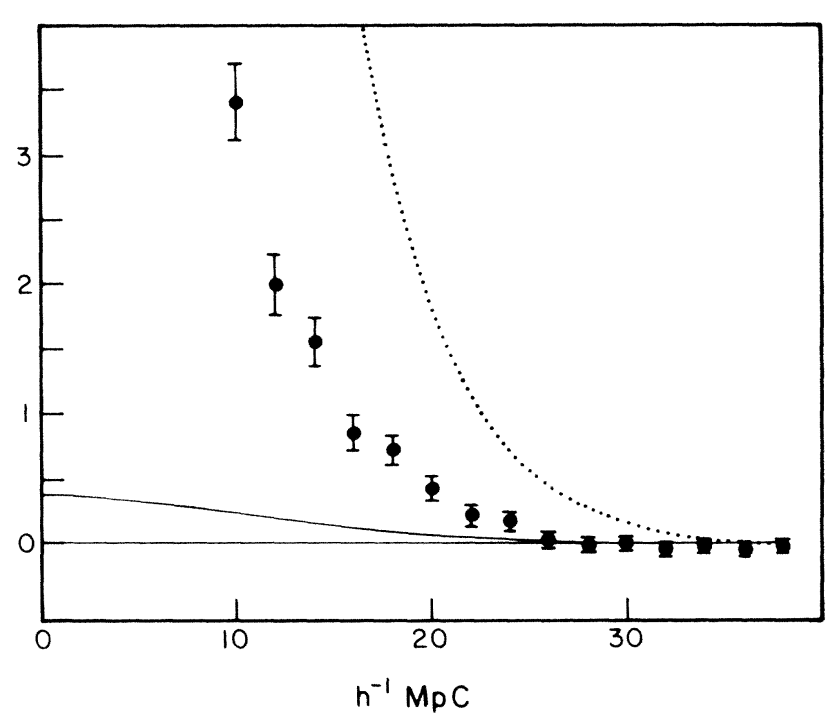

FIG. 2. Same as Fig. 1 but with an averaging radius of $9 h^{-1} \mathrm{Mpc}$.

prejudices in favor of $\Omega=1$. The results of Ref. 4 indicate that it is difficult to maintain this simple model for both galaxies and rich clusters of galaxies. However, we believe that this model is probably more reasonable for the rich clusters. Because of the much larger masses and distances involved, the ability of nonlinear gravitational evolution and other physics (such as shocks) to redistribute significantly the protolumps may be far less effective in the case of seeds of rich clusters than for the seeds of galaxies themselves.

Irrespective of the physics of galaxy formation, we have a potentially significant conclusion for the model of rich clusters as unusually large fluctuations in the primordial mass distribution. If that distribution is Gaussian and has a Zel'dovich spectrum, then the refinement of restricting the model to high peaks in that distribution implies that the rich-cluster correlation falls to negligible values significantly faster than the amplified Zel'dovich spectrum itself. If future observations support the present indications of positive rich-cluster correlations out to $30 h^{-1} \mathrm{Mpc}$, then some element of the model must be wrong.

This work was supported in part by the U. S. Department of Energy under Contracts No. DEAC-03-81ER40050 and No. DE-AT03-83-ER31118. One of us (S.O.) is a Bantrell Fellow. Another of us (M.B.W.) is an Alfred P. Sloan Fellow and a U. S. Department of Energy Outstanding Junior Investigator (under Contract No. DE-FG03-84ER40172).

${ }^{1} \mathrm{M}$. Hauser and P. Peebles, Astrophys. J. 185, 757 (1973); N. Bachall and R. Soniera, Astrophys. J. 270, 20 (1983); A. Klypin and A. Kopylov, Pis'ma Astron. Zh. 9, 75 (1983) [Sov. Astron. Lett. 9, 41 (1983)].

${ }^{2}$ N. Kaiser, Astrophys. J. 284, L9 (1984).

${ }^{3}$ H. Politzer and M. Wise, Astrophys. J. 285, L1 (1984).

$4 \mathrm{~J}$. Bardeen, J. Bond, N. Kaiser, and A. Szalay, Astrophys. J. (to be published).

$5 \mathrm{~J}$. Peacock and A. Heavens, Mon. Not. Roy. Astron. Soc. 217, 805 (1985).

${ }^{6}$ Y. Zel'dovich, Mon. Not. Roy. Astrom. Soc. 160, 1p (1972); E. Harrison, Phys. Rev. D 1, 2726 (1970); P. Peebles and J. Yu, Astrophys. J. 162, 815 (1970).

${ }^{7}$ This formula was derived previously in Ref. 4. They also consider terms subdominant in the threshold $t$. See also R. Adler, The Geometry of Random Fields (Wiley, New York, 1981); A. Doroshkevich, Astrophysics (Engl. Transl.) 6, 320 (1970).

${ }^{8}$ In general, Eq. (4) is difficult to evaluate without use of such an expansion because it involves the determinants and inverses of large matrices.

9P. Peebles, Astrophys. J. 263, L1 (1982); L. Blumenthal and J. Primak, in Formation and Evolution of Galaxies and Large Structures in the Universe-Third Moriond Astrophysics Meeting, edited by Jean Audouze and Jean Tran Thanh Van (Reidel, Dordrecht, The Netherlands, 1984); J. Bond and L. Efstathiou, Astrophys. J. 285, L45 (1984); J. Bardeen, unpublished. 